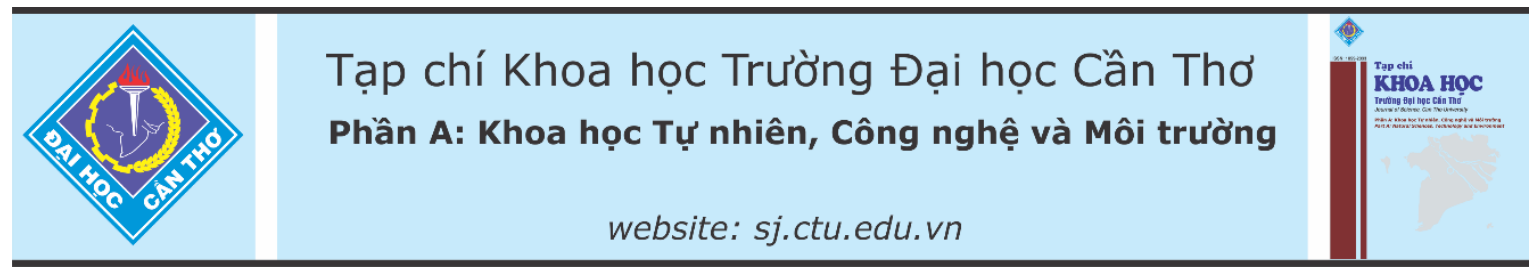

DOI:10.22144/ctu.jvn.2021.137

\title{
NANOTEBUCONAZOLE: ĐIỀU CHẾ TRONG MÔI TRƯờNG NƯỚC VÀ KHẢO SÁT HIỆU QUẢ KHÁNG NẤM BỆNH GÂY HẠI TRÊN CÂY TRỔNG
}

\author{
Phạm Quốc Yên ${ }^{1 *}$ và Trần Quốc Tuấn ${ }^{2}$ \\ ${ }^{1}$ Khoa Hóa học, Truờng Đại học Khoa học Tự nhiên-Đại học Quốc gia Thành phố Hồ Chí Minh \\ ${ }^{2}$ Khoa Sinh học và Công nghệ Sinh học, Truờng Đại học Khoa học Tự nhiên-Đại học Quốc gia Thành phố \\ Hồ Chí Minh \\ *Người chịu trách nhiệm về bài viết: Phạm Quốc Yên (email: phqyen@ gmail.com)
}

\section{Thông tin chung:}

Ngày nhận bài: $28 / 05 / 2021$

Ngày nhận bài sưa: 13/08/2021

Ngày duyệt đăng: 29/10/2021

\section{Title:}

Nanotebuconazole: Synthesis in aqueous solution and study on anti-fungal efficiency in crop

\section{Tù khóa:}

Kháng nấm, nanotebuconazole, nhũ tuong nano, polyethylene glycol, thuốc bảo vệ thưc vật

\section{Keywords:}

Anti-fungal, nanotebuconazole, nanoemulsion, polyethylene glycol, pesticide

\begin{abstract}
Tebuconazole was dispersed in aqueous solutions of polyethylene glycol (PEG) and water in a desired mass fraction with a presence of dichloromethane (DCM). The mixture was then evaporated at $40^{\circ} \mathrm{C}$ under a pressure of 480 mbar to remove DCM and form nanotebuconazole. Nanotebuconazole particles size range is $27-35 \mathrm{~nm}$ with average size of $29 \mathrm{~nm}$ that was measured by dynamic light scattering (DLS) and the results showed that the ratio of $\mathrm{PEG}: \mathrm{H}_{2} \mathrm{O} 4: 1$ is the most favorable for nanoparticle formation. In vitro tests using minimum inhibitory concentration (MIC) method illustrated that anti-fungal and antibacterial effectiveness of nanotebuconzole was demonstrated twofold increase compared with that of commercial tebuconazole for Ralstonia solanacearum, Fusarium ambrosium and Rhizoctonia solani and fourfold increase for Phytophthora capsici with MIC value are 50 ppm and $25 \mathrm{ppm}$ respectively. Nanotebuconazole prepared in this study fully meets the standards of pesticides according to Vietnamese National Standards 9482:2012 (TCVN 9482:2012).
\end{abstract}

\section{TÓM TẮT}

Tebuconazole đurợc phân tán trong hồn hơp polyethylene glycol (PEG) và nuớc theo một tỉ lệ xác định với sụ hiện diện của dichloromethane (DCM). Hồn hợp sau đó được làm bay hoi ở $40^{\circ} \mathrm{C}$ duoới áp suất 480 mbar để loại bỏ DCM và hình thành nanotebuconazole. Kích thuoóc hạt nanotebuconazole được xác định trong khoảng $27-35 \mathrm{~nm}$ với kích thước trung bình là $29 \mathrm{~nm}$ bằng kĩ thuật tán xạ ánh sáng động (DLS) và kết quả nghiên cứu cho thấy tì lẹ PEG: $\mathrm{H}_{2} \mathrm{O}$ 4:1 là thuận lợi nhất cho quá trình hình thành hạt nhũ nano. Các thư nghiệm in vitro bằng phuoong pháp MIC (minimum inhibitory concentration) chúng minh rằng dạng nanotebuconazole cho hiệu quả kháng khuẩn, kháng nấm mạn gấp 2 lần dạng thuơng mại trên các chủng Ralstonia solanacearum, Fusarium ambrosium và Rhizoctonia solani và gấp 4 lần trên chủng Phytophthora capsici, với nồng độ ức chế tối thiểu lần luợt là 50 ppm và 25 ppm. Chế phẩm nanotebuconazole được đánh giá chất luợng đáp ưng đầy đủ tiêu chuẩn thuốc bảo vệ thực vật theo Tiêu chuẩn Việt Nam 9482:2012 (TCVN 9482:2012). 


\section{GIỚI THIỆ}

Trong lĩnh vực nông nghiệp, thuốc bảo vệ thực vật đã và đang đóng vai trò then chốt trong việc phòng trừ sâu bệnh gây hại, bảo vệ và nâng cao năng suất cây trồng hàng trăm năm qua. Tuy nhiên, bên cạnh những lợi ích mà chúng mang lại thì độc tố, dư lượng và những tác động tiêu cực đến sức khỏe con người cũng như hệ sinh thái luôn là vấn đề nóng cần có giải pháp khắc phục. Hiện nay với sự tiến bộ của khoa học kĩ thuật, ngành thuốc bảo vệ thực vật cũng có sự phát triển vượt bậc và ngày càng hướng đến yếu tố môi trường. Những hoạt chất có nguồn gốc sinh học đã được nghiên cứu và đưa vào trong sản xuất để thay thế các hoạt chất hóa học như abamectin (Dybas et al., 1989), emamectin (Isaac et al., 2002),... đã dần phát huy tác dụng tác động chọn lọc và ít tác động tới môi trường. Một hướng nghiên cứu mới đó là ứng dụng công nghệ nano trong sản xuất các hoạt chất nhằm mục đích tăng hoặc giữ nguyên hoạt tính mà liều lượng sử dụng ít hơn so với dạng thương mại. Có nhiều phương pháp để tổng hợp các hoạt chất dạng nano như phân tán trên nền của polymer (polymer based): imidacloprid (Adak et al., 2012; Kaushik et al., 2013), thiamethoxam (Loha et al., 2011, 2012; Pankaj et al., 2012), carbofuran (Sarkar et al., 2012) hoặc chế tạo dạng hạt nano lipid: deltamethrin (Nguyen et al., 2012) hay dạng hệ nhũ tương nano: permethrin (Kumar et al., 2013), glyphosate (Jiang et al., 2012). Theo Kah et al. (2013), thuốc bảo vệ thực vật dưới dạng nano bao gồm microemulsion, nanoemulsions, nanodispersion, có kích thước hạt nhũ dưới 1000 $\mathrm{nm}$. Trong đó, dạng nano (nanoformulation) được quan tâm nhiều nhất. Kích thước nhỏ góp phần tăng hoạt lực của thuốc được chứng minh qua nhiều nghiên cứu đã công bố. Roy et al. (2018) đã tổng hợp thành công nano hexaconazole (N-hexa) một chât trong họ triazole, kích thước hạt được phân tích bằng kính hiển vi điện tử quét (SEM), kết quả so sánh hình thái phát triển của nấm in vitro cho thấy $\mathrm{N}$-hexa hiệu quả hơn nhiều so với hexaconazole thương mại. Tebuconazole [(RS)-1-p-chlorophenyl4,4-dimethyl-3-(1H-1,2,4-triazol-1-ylmethyl)

pentan-3-ol] là hoạt chất diệt nấm bệnh thuộc nhóm triazole. Đích tác dụng của tebuconazole và nhóm triazole là ức chế sinh tổng hợp ergosterol, là chất cần thiết cho sự hình thành thành tế bào của nấm. Trong đó, các hoạt chất ức chế quá trình khử hóa nhóm methyl chống lại phản ứng oxi hóa trung gian cytochrome P450 dẫn đến bất hoạt quá trình sinh tổng hợp ergosterol (Sancholle et al., 1984). Tebuconazole có tác dụng diệt nấm phổ rộng trên lá, trong đất nhưng khó tan trong nước, do đó các chế phẩm chứa tebuconazole hiện nay trên thị trường được chế tạo bằng phương pháp nhũ hóa cần nhiều dung môi hữu cơ thân dầu kém thân thiện với môi trường. Do đó, trong nghiên cứu này, chế phẩm nanotebuconazole được tổng hợp bằng phương pháp phân tán trong môi trường nước kết hợp với polyethylene glycol (PEG) được biết đến là những dung môi xanh, để giảm thiểu sự tác động của thuốc đến sức khỏe con người cũng như hệ sinh thái.

\section{THỰC NGHIÊM}

\subsection{Vật liệu và thiết bị}

Tebuconazole thương mại, polyethylene glycol (PEG), dichlormethane (DCM), polysorbate 80 (TW80) từ Xilong Scientific Co., Ltd, Trung Quốc.

Thiết bị tán xạ ánh sáng động (DLS-Horiba SZ100 Nanoparticle Analyzer, Horiba, Nhật Bản) dùng để xác định kích thước hạt nanotebuconazole phân tán. Thiết bị vận hành dưới góc $90^{\circ}$ và nhiệt độ $24^{\circ} \mathrm{C}$. Hàm lượng tebuconazole được kiểm tra bằng phương pháp sắc kí khí, đầu dò ion hóa ngọn lửa (GC-FID) sử dụng cột mao quản HP-5MS, $(30 \mathrm{~m} \mathrm{x}$ $0,25 \mathrm{~mm} \times 0,25 \mu \mathrm{m})$, nhiệt độ đầu dò $320^{\circ} \mathrm{C}$, tốc độ dòng khí $\mathrm{H}_{2} 35 \mathrm{~mL}$.phút ${ }^{-1}$, tốc độ khí mang He 1,7 $\mathrm{mL}$.phút ${ }^{-1}$ tốc độ dòng không khí duy trì ngọn lửa 420 mL.phút $\mathrm{t}^{-1}$.

\section{2. Điều chế nanotebuconazole}

Qui trình điều chế: Cân $5 \mathrm{~g}$ tebuconazole kĩ thuật vào trong bình nón có dung tích $100 \mathrm{~mL}$, hòa tan tebuconazole bằng $50 \mathrm{~mL} \mathrm{DCM}$ và $10 \mathrm{~mL}$ polysorbate 80 . $\mathrm{PEG}$ và $\mathrm{H}_{2} \mathrm{O}$ lần lượt được cân và cho vào ống teflon có nắp đậy theo tỉ lệ khối lượng $(\mathrm{w} / \mathrm{w})$ thay đổi lần lượt là $9: 1,6: 1,4: 1,3: 1$ và $1: 1$, đổ mạnh dung dịch tebuconazole từ bình nón vào ống teflon, đậy kín và lắc hỗn hợp bằng máy lắc Vortex ở $2000 \mathrm{rpm}$ trong 10 phút. Sau đó, dung môi $\mathrm{DCM}$ bị loại bằng cô quay chân không ở $40^{\circ} \mathrm{C}$, áp suất 480 mbar thu được sản phẩm cuối cùng (Hình 1). Sự ảnh hưởng của tỉ lệ $\mathrm{PEG}: \mathrm{H}_{2} \mathrm{O}$ đến độ bền của nanotebuconazole cũng được khảo sát trong nghiên cứu này.

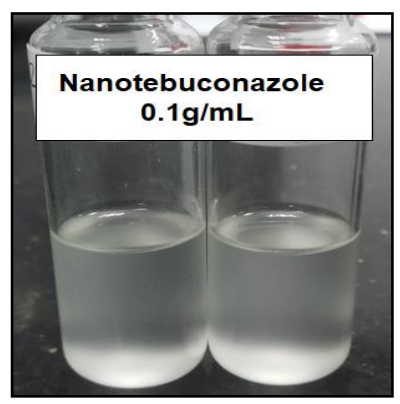

Hình 1. Nanotebuconazole 


\subsection{Khảo sát hiệu quả kháng khuẩn và kháng nấm của nanotebuconazole}

\subsubsection{Phurong pháp khảo sát hoạt tính kháng nấm bằng phuoong pháp đục lỗ thạch}

Các chủng vi sinh vật được khảo sát trong nghiên cứu này bao gồm: 1. Ralstonia solanacearum, 2. Xanthomonas oryzae pv. Oryzae, 3. Bacillus cereus ATCC 11778, 4. Phytophthora capsici, 5. Fusarium ambrosium, 6. Rhizoctonia solani.

Hoạt hóa chủng nấm thử nghiệm bằng môi trường cao thịt peptone trong $24 \mathrm{~h}$. Sau đó, mật độ chủng nấm được xác định bằng phương pháp đo mật độ quang ở bước sóng $660 \mathrm{~nm}$ và điều chỉnh về mật độ chủng nấm trong khoảng là khoảng 106- 107 CFU.ml ${ }^{-1}$. Chủng nấm thử nghiệm được cấy lên môi trường cao thịt peptone. Thử nghiệm khả năng kháng nấm của nanotebuconazole bằng cách hút 40 $\mu 1$ dung dịch mẫu thử nồng độ khác nhau trong nước cất vô trùng vào các giếng trên đĩa thạch đã trải chủng nấm thử nghiệm. Sử dụng chloramphenicol $(0,2 \mathrm{ppm})$ dùng làm chứng dương. Dùng nước cất vô trùng làm chứng âm. Sau 24 giờ, kết quả được ghi nhận bằng hình ảnh và đường kính vòng vô khuẩn (ĐKVK).

ĐKVK được tính theo công thức:

ĐKVK = ĐKVK mẫu thử - ĐKVK giếng

2.3.2. Định luợng khả năng kháng khuẩn, kháng nấm của nanotebuconazole bằng phương pháp MIC

MIC của mẫu thử được khảo sát bằng phương pháp pha loãng trên phiến vi lượng 96 giếng. Mỗi giếng gồm $100 \mu \mathrm{l}$ môi trường có khuẩn và $100 \mu \mathrm{l}$ mẫu thử pha loãng trong môi trường. Mẫu được pha loãng theo dãy nồng độ khác nhau (0-500ppm). Mẫu được ủ ở $37^{\circ} \mathrm{C}$ trong 16-24 giờ. Sau 24 giờ, 20 $\mu \mathrm{L}$ thuốc thử resazurin $0,01 \%$ được cho vào mỗi giếng; quan sát sự thay đổi màu, ghi nhận giá trị MIC.

\section{KẾT QUẢ VÀ THẢO LUẬN}

\subsection{Sự ảnh hưởng của tỉ lệ PEG: $\mathrm{H}_{2} \mathrm{O}$}

Nước đóng vai trò là môi trường phân tán, $\mathrm{PEG}$ đóng vai trò như mạng liên kết lưu giữ các hạt nanotebuconazole không kết hợp vào nhau, do đó tỉ lệ PEG: $\mathrm{H}_{2} \mathrm{O}$ là yếu tố rất quan trọng đến độ bền của chế phẩm. Trong nghiên cứu này, $\mathrm{PEG}: \mathrm{H}_{2} \mathrm{O}(\mathrm{w} / \mathrm{w})$ được khảo sát theo từng tỉ lệ khác nhau, dựa trên yếu tố phân tách pha sau các khoảng thời gian khảo sát để kiểm tra độ bền của sản phẩm. Kết quả thu được trình bày ở Bảng 1 .

Bảng 1. Độ bền nhũ theo thời gian

\begin{tabular}{ccccc}
\hline $\begin{array}{c}\text { Tỉ lệ } \\
\text { PEG:H2O } \\
\text { (w/w) }\end{array}$ & \multicolumn{4}{c}{$\begin{array}{c}\text { Độ bền sau khoảng thò̀i } \\
\text { gian khảo sát (giờ) }\end{array}$} \\
\cline { 2 - 5 } & $\mathbf{2 4}$ & $\mathbf{7 2}$ & $\mathbf{2 4 0}$ & $\mathbf{7 2 0}$ \\
\hline $9: 1$ & + & + & + & + \\
$6: 1$ & + & + & + & + \\
$4: 1$ & + & + & + & + \\
$3: 1$ & + & - & - & PL \\
$1: 1$ & PL & PL & PL & PL \\
\hline
\end{tabular}

Ghi chú: (+) bền, (-) kém bền, (PL) phân lớp hoàn toàn.

Kết quả cho thấy độ bền của nanotebuconazole giảm khi tăng tỉ lệ nước, điều này có thể giải thích được vì tebuconazole ít tan được trong nước, do đó hàm lượng nước cao, các hạt nano có khuynh hướng kết tụ lại với nhau, khó bảo quản được sản phẩm. Trong nghiên cứu này tỉ lệ phù hợp nhất là $4: 1$ đáp ứng được độ bền trong hơn 720 giờ.

\subsection{Xác định kích thước nanotebuconazole}

Kết quả phân tích kích thước hạt bằng DLS cho thấy nanotebuconazole tổng hợp trong nghiên cứu này có kích thước khá đồng nhất dao động từ $27-$ $35 \mathrm{~nm}$ với kích thước trung bình $29 \mathrm{~nm}$. (Hình 2A), Hình 2B thể hiện thời gian trễ trong phép đo DLS, ý nghĩa của đường biểu diễn càng gấp khúc càng hẹp kích thước hạt càng nhỏ. Có thể kết luận rằng, tebuconazole có thể phân tán được dạng nano trong môi trường nước, nhờ sự kết hợp của $\mathrm{PEG}$ và $\mathrm{TW} 80$. 

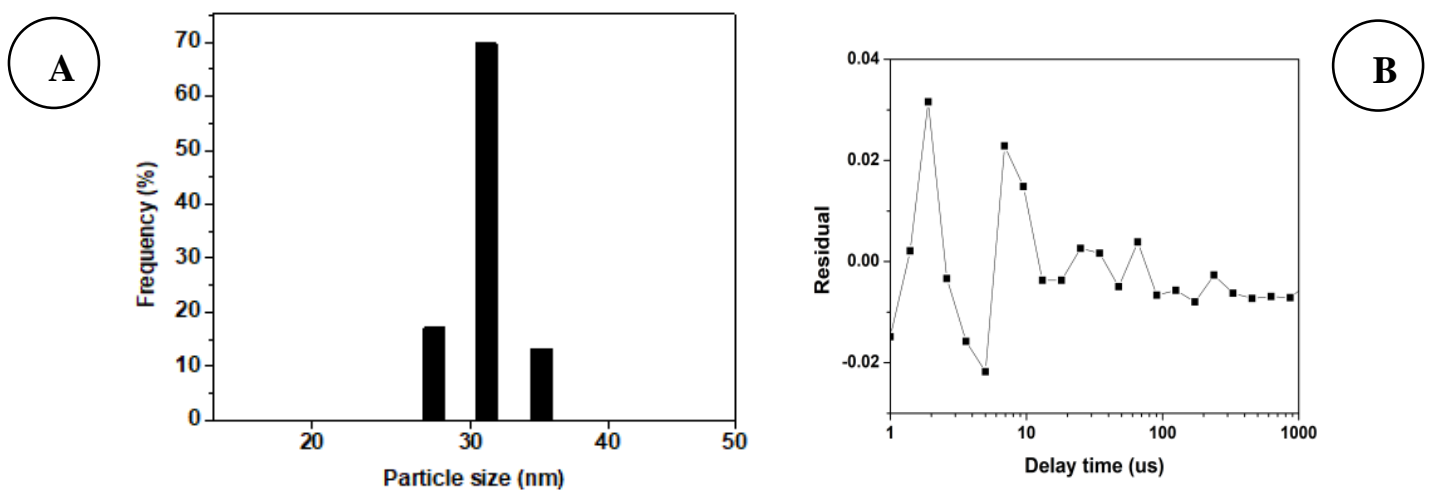

Hình 2. Phân bố kích thước $(\mathrm{A})$, và biểu diễn thời gian trễ $(\mathrm{B})$ bằng kĩ thuật DLS

3.3. Hoạt tính kháng khuẩn và nấm của nanotebuconazole

\subsubsection{Phuơng pháp khảo sát hoạt tính kháng nấm bằng phương pháp đục lỗ thạch}

Kết quả thu được trình bày ở Bảng 2 và Hình 3 . tính mạnh hơn dạng thương mại ở từng nồng độ khảo sát khác nhau trên từng chủng vi sinh vật nhất định.

Kêt quả chứng minh rằng nanotebuconazole có hoạt

Bảng 2. Kết quả thử nghiệm hoạt tính ức chế sự sinh trưởng của vi khuẩn và nấm của nanotebuconazole và tebuconazole thương mại ở các nồng độ thử nghiệm khác nhau

\begin{tabular}{|c|c|c|c|c|}
\hline \multirow{3}{*}{$\begin{array}{l}\text { Mẫu thử } \\
\text { ( } \mu \text { g/giếng) }\end{array}$} & \multicolumn{4}{|c|}{ ĐKVK $(\mathrm{mm})$} \\
\hline & \multicolumn{2}{|c|}{ Ralstonia Xanthomonas oryzae } & \multirow{2}{*}{$\begin{array}{r}\text { Bacillus cereus } \\
\text { ATCC } 11778\end{array}$} & \multirow{2}{*}{$\begin{array}{r}\text { Phytophthora } \\
\text { capsici }\end{array}$} \\
\hline & solanacearum & pv. Oryzae & & \\
\hline Nanotebuconazole- 10 & $24 \pm 0.2$ & $6,0 \pm 0,0$ & $6,0 \pm 0,0$ & $24,0 \pm 0,1$ \\
\hline Nanotebuconazole- 100 & $35 \pm 0.2$ & $6,0 \pm 0,0$ & $12,5 \pm 0,1$ & $28,0 \pm 0,1$ \\
\hline Tebuconazole- $\quad 10$ & $6,0 \pm 0.0$ & $6,0 \pm 0,0$ & $6,0 \pm 0,0$ & $12,5 \pm 0,2$ \\
\hline Tebuconazole- $\quad 100$ & $6,0 \pm 0.0$ & $6,0 \pm 0,0$ & $8,0 \pm 0,1$ & $30,0 \pm 0,1$ \\
\hline Chloramphenicol- 0,2 & $13,0 \pm 0.2$ & $24,0 \pm 0,2$ & $26,0 \pm 0,1$ & $28 \pm 0,3$ \\
\hline
\end{tabular}

*Đuòng kính giếng thạch: $6 \mathrm{~mm}$, ĐKVK = 6 mm tương úng không kháng
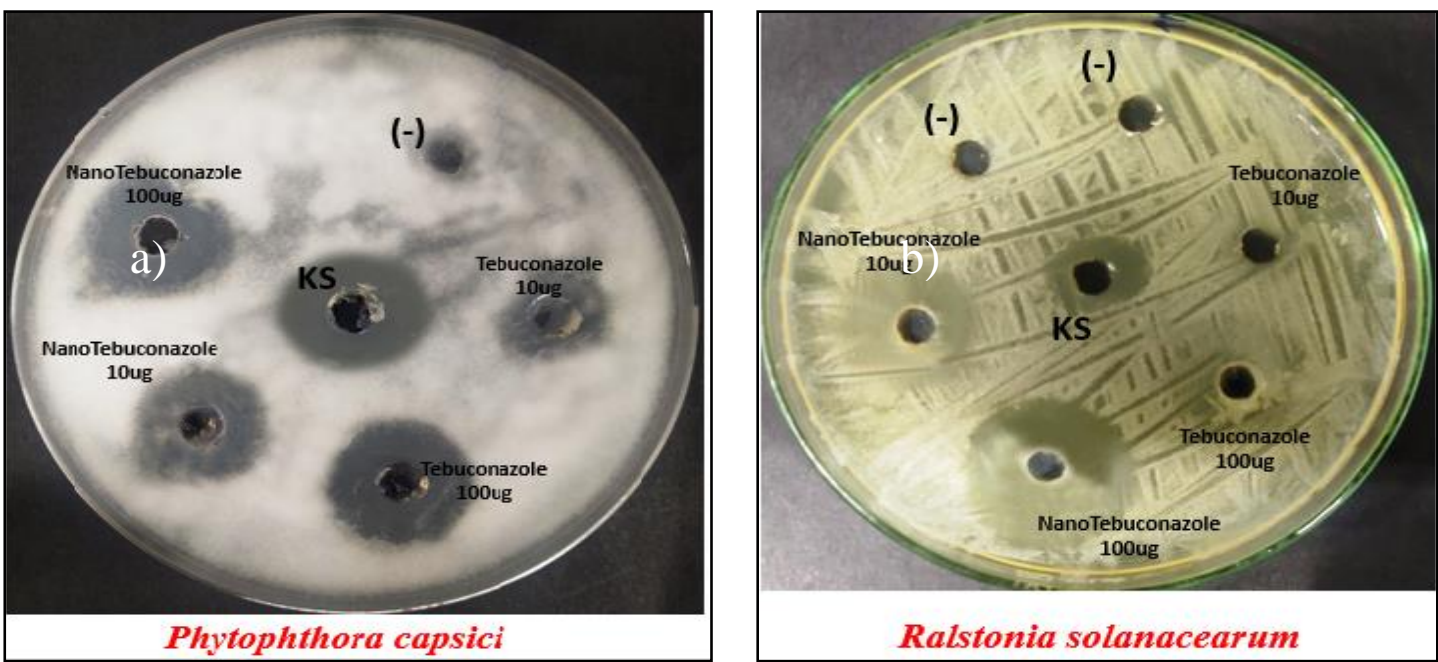

Hình 3. Vòng vô khuẩn của nanotebuconazole và tebuconazole thương mại ở các nồng độ khác nhau đối với 2 chủng vi sinh vật a) Phytophthora capsici và b) Ralstonia solanacearum 
Cụ thể trên chủng vi khuẩn Ralstonia solanacearum nanotebuconazole cho khả năng ức chế sinh trưởng (ĐKVK $24 \pm 0,2$ ở nồng độ 10 $\mu \mathrm{g} / \mathrm{giế}$ g) trong khi tebuconazole thương mại hoàn toàn không có hoạt tính. Trên chủng nấm Phytophthora capsici khả năng ức chế của nanotebuconazole tốt hơn tenuconazole thương mại ở nồng độ thấp $10 \mu \mathrm{g} / \mathrm{giế}$ g (ĐKVK nanotebuconazole là $24 \pm 0,2 \mathrm{~mm}$ gấp 2 lần giá trị của tebuconazole $12,5 \pm 0,2$ ) và tương đương ở nồng độ $100 \mu \mathrm{g} /$ giếng có ĐKVK lần lượt là $28 \pm 0,1$ $\mathrm{mm}$ và $30 \pm 0,1 \mathrm{~mm}$.
3.3.2. Định lương khả năng kháng khuẩn, kháng nấm của nanotebuconazole bằng phuoong pháp MIC

Từ kết quả khảo sát (Bảng 3) cho thấy nanotebuconazole cho hoạt lực mạnh hơn dạng thương mại gấp hai lần trên các chủng Ralstonia solanacearum, Fusarium ambrosium, Rhizoctonia solani và gấp bốn lần trên chủng Phytophthora capsici. Điểu đó chứng minh rằng, kích thước rất bé của các hạt nhũ nanotebuconazole tổng hợp làm tăng sự khuếch tán, thẩm thấu dẫn đến tăng hoạt tính sinh học của hoạt chất. Điều này phù hợp với xu hướng dự đoán của nghiên cứu này.

Bảng 3. Giá trị MIC của nanotebuconazole và tebuconazole trên các chủng vi sinh vật khác nhau

\begin{tabular}{lcccc}
\hline \multirow{2}{*}{ Mẫu thử } & \multicolumn{4}{c}{ MIC (ppm) } \\
\cline { 2 - 5 } & $\begin{array}{c}\text { Ralstonia } \\
\text { solanacearum }\end{array}$ & $\begin{array}{c}\text { Fusarium } \\
\text { ambrosium }\end{array}$ & Rhizoctonia solani & $\begin{array}{c}\text { Phytophthora } \\
\text { capsici }\end{array}$ \\
\hline Nanotebuconazole & 50 & 50 & 50 & 25 \\
Tebuconazole & 100 & 100 & 100 & 100 \\
Chloramphenicol & 0,2 & 0,2 & 0,2 & 0,2 \\
\hline
\end{tabular}

\section{4. Đánh giá tính chất hóa lý của chế phẩm}

Chế phẩm nanotebuconazole được tạo thành có đặc tính của thuốc bảo vệ thực vật dạng nhũ dầu EC, tuy nhiên sự khác biệt lớn nhất là ở dung môi pha chế, dùng dung môi nước và $P E G$ thay thế các dung môi hữu cơ. Để đánh giá chất lượng chế phẩm theo
TCVN 9482:2012, độ bền lưu trữ trên các chỉ tiêu hóa lý: độ bền nhũ, độ tạo bọt, hàm lượng tebuconazole sau khi ủ ở $0^{\circ} \mathrm{C}$ và $54^{\circ} \mathrm{C}$ sau 2 tuần được lựa chọn. Kết quả cho thấy chế phẩm hoàn toàn đạt được các chỉ tiêu theo tiêu chuẩn TCVN 9482:2012. Kết quả được thể hiện trong Bảng 4.

Bảng 4. Kết quả đánh giá chất lượng chế phẩm nanotebuconazole

\begin{tabular}{llccc}
\hline Chỉ tiêu & Yêu cầu theo & Ban đầu & Mẫu ủ ở 0 & Mẫu ủ ở $\mathbf{5 4}^{\circ} \mathbf{C}$ \\
\hline Độ tạo bọt $(\mathrm{mL})$ & Thề tích bọt $\leq 60 \mathrm{~mL}$ & 2 & 5 & 8 \\
Độ tự nhũ ban đầu & Hoàn toàn & Hoàn toàn & Hoàn toàn & Hoàn toàn \\
Độ bền nhũ sau 0,5 giờ & Thể tích lớp kem $\leq 2 \mathrm{~mL}$ & 0 & 0 & 0 \\
Độ bền nhũ sau 2 giờ & Thể tích lớp kem $\leq 4 \mathrm{~mL}$ & 0 & 0 & 0 \\
Hàm lượng hoạt chất $(\%)$ & Sai lệch cho phép $\pm 15 \%$ & 8,62 & 8,88 & 9,02 \\
\hline
\end{tabular}

\section{KẾT LUẬN}

Nanotebuconazole lần đầu được điều chế dưới dạng phân tán trong dung môi nước, tỉ lệ $\mathrm{H}_{2} \mathrm{O}: \mathrm{PEG}$ được kiểm soát $1: 4$ để được sản phẩm bền không bị phân tách pha, kích thước hạt tạo thành rất nhỏ từ 27 - $35 \mathrm{~nm}$ góp phần làm tăng hoạt tính sinh học vốn có của tebuconazole lên 2 lần đối với Ralstonia solanacearum, Fusarium ambrosium và Rhizoctonia solani; đặc biệt nanotebuconazole cho hoạt tính kháng chủng nấm Phytophthora capsici (chủng nấm gây bệnh chết nhanh rất nghiêm trọng trên cây hồ tiêu) gấp 4 lần so với dạng thương mại. Uuu điểm của phương pháp điều chế này: (i) đơn giản, dễ thực hiện, (ii) tăng hoạt tính sinh học, (iii) giảm thiểu ô nhiễ்m môi trường khi thay thế các dung môi hữu cơ độc hại truyền thống trong công nghệ sản xuất thuốc bảo vệ thực vật bằng dung môi thân thiện với môi trường. Khi đánh giá chất lượng chế phẩm tạo thành theo TCVN 9482:2012, chế phẩm đạt yêu cầu trên tất cả chỉ tiêu. Do đó, nghiên cứu này đáng được thực hiện trên các loại hoạt chất khác cũng như áp dụng khảo nghiệm trên cây trồng để góp phần sớm đưa những sản phẩm bảo vệ thực vật "xanh" vào thực tế.

\section{LỜI CẢM TẠ}

Học viên cao học Phạm Quốc Yên được tài trợ bởi chương trình học bổng đào tạo thạc sĩ, tiến sĩ trong nước của Quỹ Đổi mới sáng tạo Vingroup (VINIF), Viện Nghiên cứu Dữ liệu lớn (VinBigdata) thuộc tập đoàn Vingroup, mã số 2020.ThS48. 


\section{TÀI LIỆ THAM KHẢO}

Adak, T., Kumar, J., Shakil, N. A., \& Walia, S. (2012). Development of controlled release formulations of imidacloprid employing novel nano-ranged amphiphilic polymers. $J$ Environ Sci Health B, 47(3), 217-25.

Dybas, R. A. (1989). Abamectin Use in Crop Protection. In C. C William (Eds). Ivermectin and Abamectin (pp 287-310). Sringer.

Isaac, I., Svetlana, K., \& Rami, H. A. (2002). Emamectin, a novel insecticide for controlling field crop pests. Pest Management Science, 58(11), 1091-1095.

Jiang, L. C., Basri, M., Omar, D., Rahman, M. B. A., Salleh, A. B., Rahman, R. N. Z. R.A., \& Selamat, A. (2012). Green nano-emulsion intervention for water-soluble glyphosate isopropylamine (IPA)formulations in controlling Eleusine indica (E-indica). Pesticide Biochemistry and Physiology, 102(1), 19-29.

Kah, M., Beulke, S., Tiede, K., \& Hofmann, T. (2013). Nano-pesticides: state of knowledge, environmental fate and exposure modelling. Critical Reviews in Environmental Science and Technology, 43, 1823-1867.

Kaushik, P., Shakil, N. A., Kumar, J., Singh, M. K., \& Yadav, S. K. (2013). Development of controlled release formulations of thiram employing amphiphilic polymers and their bioefficacy evaluation in seed quality enhancement studies. Journal of Environmental Science and Health, Part B, 48(8), 677-85.

Kumar, R. S. S., Shiny, P. J., Anjali, C. H., Jerobin, J., Goshen, K. M., \& Magdassi, S. (2013). Distinctive effects of nano-sized permethrin in the environment. Environmental Science and Pollution Research, 20(4), 2593-602.
Loha, K. M., Shakil, N. A., Kumar, J., Singh, M. K., Adak, T.\& Jain, S. (2011). Release kinetics of beta-cyfluthrin from its encapsulated formulations in water Journal of Environmental Science and Health, Part B, 46(3), 201-6.

Loha, K. M., Shakil, N. A., Kumar, J., Singh, M., \& Srivastava, C. (2012). Bio-efficacy evaluation of nanoformulations of beta-cyfluthrin against Callosobruchus maculatus (Coleoptera: Bruchidae). Journal of Environmental Science and Health, Part B, 47(7), 687-91.

Nguyen, H. M., Hwang, I. C., Park, J. W., \& Park, H. J. (2012). Enhanced payload and photoprotection for pesticides using nanostructured lipid carriers with corn oil as liquid lipid. Journal of Microencapsulation, 29(6), 596-604.

Pankaj Shakil, N. A., Kumar, J., Singh, M. K., \& Singh, K. (2012). Bioefficacy evaluation of controlled release formulations based on amphiphilic nano-polymer of carbofuran against Meloidogyne incognita infecting tomato. Journal of Environmental Science and Health, Part B, 47(6), 520-8.

Roy, I., Thapa, M., \& Goswami, A. (2018). Nanohexaconazole: synthesis, characterisation and efficacy of a novel fungicidal nanodispersion. IET Nanobiotechnology, 12(6), 684-688.

Sancholle, M., Weete, J., \& Montani, C. (1984). Effect of triazoles on fungi I: Growth and cellular permeabilit. Pesticide Biochemistry and Physiology, 21(1), 31-44.

Sarkar, D. J., Kumar, J., Shakil, N. A., \& Walia, S. (2012). Release kinetics of controlled release formulations of thiamethoxam employing nanoranged amphiphilic PEG and diacid based block polymers in soil. Journal of Environmental Science and Health, Part A, 47(11), 1701-1712. 